\title{
SINGULAR CONTINUOUS SPECTRUM IS GENERIC
}

\author{
R. DEL RIO, S. JITOMIRSKAYA, N. MAKAROV, AND B. SIMON
}

\begin{abstract}
In a variety of contexts, we prove that singular continuous spectrum is generic in the sense that for certain natural complete metric spaces of operators, those with singular spectrum are a dense $G_{\delta}$.
\end{abstract}

In the spectral analysis of various operators of mathematical physics, a key step, often the hardest, is to prove that the operator has no continuous singular spectrum, that is, that the spectral measures for the operators have only pure point and absolutely continuous parts. Examples are the absence of such spectrum for $N$-body Schrödinger operators $[3,19]$ and for the one-dimensional random models $[12,7,8,24,18]$.

Our goal here is to announce results that show that a singular continuous spectrum lies quite close to many operators by proving it is often generic in the Baire sense. Detailed proofs and further results will appear in three papers: one for general operators [22], one for rank-one perturbations [6], and one for almost-periodic Schrödinger operators [23].

Precursors of our results include work on generic ergodic processes [15, 21] and on special energies for Schr̈odinger operators/Jacobi matrices $[11,4,5]$. Gordon [13,14] independently (and presumably, before us) proved Theorem 5. His method of proof is very different from ours.

Recall that the Baire category theorem implies that if $X$ is a complete metric space, a countable intersection of dense $G_{\delta}$ is still a dense $G_{\delta}$ and that if $X$ is perfect, then any dense $G_{\delta}$ has uncountable intersection with any open ball.

Our first two results are for one-body Schrödinger operators and for the "generic Anderson model".

Theorem 1 [22]. Let $C_{\infty}\left(\mathbb{R}^{\nu}\right)$ denote the continuous functions on $\mathbb{R}^{\nu}$ vanishing at infinity in the $\|\cdot\|_{\infty}$ norm. Then for a dense $G_{\delta}$ of $V \in C_{\infty}\left(\mathbb{R}^{\nu}\right),-\Delta+V$ has purely singular continuous spectrum on $(0, \infty)$.

Remarks. 1. If $V(x)=0\left(|x|^{-1-\epsilon}\right)$ at infinity, it is known [1, 20] that $-\Delta+V$ has absolutely continuous spectrum on $(0, \infty)$ with a possible set of eigenvalues.

2. There is a similar result [22] for $\left\{V \mid\left(1+x^{2}\right)^{\alpha / 2} V \in C\left(\mathbb{R}^{\nu}\right)\right\}$ with norm \|\|$V\|=\|\left(1+x^{2}\right)^{\alpha / 2} V \|_{\infty}$ so long as $\alpha<\frac{1}{2}$.

3. In one dimension, there is a similar result for Jacobi matrices [22].

Received by the editors May 3, 1993.

The first author's research was partially supported by DGAPA-UNAM and CONACYT.

This material is based upon work of the third author supported by National Science Foundation grant DMS-9207071. The government has certain rights in this material.

This material is based upon work of the first and fourth authors supported by National Science Foundation grant DMS-9101715. The government has certain rights in this material. 
Theorem 2 [22]. Let $a<b$ be fixed and let $\Omega=[a, b]^{\mathbf{Z}^{\nu}}$, functions $v: \mathbb{Z}^{\nu} \rightarrow$ $[a, b]$ with the (compact metrizable) Tychonoff topology. Given any $v$, let $h(v)$ be the Jacobi matrix on $l^{2}\left(\mathbb{Z}^{\nu}\right)$ by

$$
(h(v) u)(n)=\sum_{|j|=1} u(n+j)+v(n) u(n) .
$$

Then for a dense $G_{\delta}$ in $\Omega, h(v)$ has spectrum $[a-2 \nu, b+2 \nu]$, and the spectrum is purely singular continuous.

Remark. If $\nu=1$ or if $\nu$ is arbitrary and $b-a$ is large, it is known that if $\Omega$ is given the product measure $\underset{j \in \mathbf{Z}^{\nu}}{\mathrm{X}}(b-a)^{-1} d x_{j}$, then for a.e. $v, h(v)$ has only pure point spectrum $[7,9,8,24,2]$. So the generic Baire and generic Lebesgue behaviors are very different.

These results are not limited to Schrödinger operators.

Theorem 3 [22]. Let $X_{a}$ be the family of all self-adjoint operators, $A$, on a fixed separable Hilbert space, $\mathscr{H}$, with $\|A\| \leq a$. Give $X_{a}$ the metrizable topology of strong convergence. Then $X_{a}$ has a dense $G_{\delta}$ of operators with $\operatorname{spec}(A)=[-a, a]$, and the spectrum is purely singular continuous.

Theorem 4 [22]. Let $A$ be a fixed self-adjoint operator. Let $\mathscr{I}_{2}$ be the HilbertSchmidt operators with Hilbert-Schmidt norm. Then for a dense $G_{\delta}$ of $C$ 's in $\mathscr{I}_{2}$, the set of vectors

$$
\begin{aligned}
& \left\{\psi \mid d \mu_{(A+C)}^{\psi} \text { is purely singular continuous }\right\} \\
& \quad \cup\left\{\psi \mid(A+C) \psi=E \psi ; E \in \operatorname{spec}_{\mathrm{disc}}(A+C)\right\}
\end{aligned}
$$

span $\mathscr{H}$.

Remarks. 1. $d \mu_{D}^{\psi}$ is the spectral measure $\left(\psi, e^{i s D} \psi\right)=\int e^{i s E} d \mu_{D}^{\psi}(E)$.

2. If $\operatorname{spec}(A)$ is thin, for example, $A \equiv 0$, the vectors are the discrete eigenvectors. But if $\operatorname{spec}(A)$ contains an interval, $\operatorname{spec}(A+C)$ will have lots of singular continuous spectrum.

3. This is to be distinguished from the Weyl-von Neumann theorem [26, $27,17]$ that there are $C$ 's with $\|C\|_{2}$ arbitrarily small so that $A+C$ has only point spectrum. Here we see that generically there will be singular continuous spectrum (for $A$ suitable).

4. The result holds if $\mathscr{I}_{2}$ is replaced by $\mathscr{I}_{p}$ with $p>1$. If $A$ has no a.c. spectrum, it even holds for $\mathscr{I}_{1}$.

These four theorems are rather soft with no hard estimates. More subtle is the case of rank-one perturbations. We will consider two closely related cases:

(a) $A$ is a self-adjoint operator with cyclic vector $\varphi$; let $P$ be the projection onto $\varphi$, and let $A_{\lambda}=A+\lambda P$.

(b) Let $H$ be the differential operator $-\frac{d^{2}}{d x^{2}}+V(x)$ on $[0, \infty)$ assumed to be limit point at infinity. $H_{\theta}$ is the self-adjoint operator with boundary condition $\cos \theta u(0)+\sin \theta u^{\prime}(0)=0$.

Theorem 5 [6]. (a) Suppose $A$ has an interval $[a, b]$ in its spectrum and the spectrum there has no a.c. component. Then

(i) There is a dense $G_{\delta}, C$, in $[a, b]$ so that if $E \in C$, then $E$ is not an eigenvalue of any $A_{\lambda}$. 
(ii) For a dense $G_{\delta}, L$, of $\mathbb{R}, A_{\lambda}$ has purely singular spectrum in $[a, b]$ if $\lambda \in L$.

(b) Suppose for some $\theta_{0}, H_{\theta_{0}}$ has an interval $[a, b]$ in its spectrum and the spectrum there has no a.c. component, then

(i) There is a dense $G_{\delta}, C$, in $[a, b]$ so that if $E \in C$, then $E$ is not an eigenvalue of any $H_{\theta}$.

(ii) For a dense $G_{\delta}, L$, of $[0, \pi), H_{\theta}$ has purely singular spectrum in $[a, b]$ if $\theta \in L$.

Remarks. 1. Case (i) implies that under the hypothesis of $E \in C$, either $\lim _{x \rightarrow \infty} \frac{1}{x} \ln \left\|T_{E}(x)\right\|$ fails to exist or is 0 when $T(E)$ is the fundamental matrix for the problem. This means that for many cases where one can only prove Lyapunov behavior for a.e. $E$, there really is a set where the Lyapunov behavior fails $[11,4,5]$.

2. There are also results for general $A$ without any hypothesis on $\operatorname{spec}(A)$ or absolute continuous spectrum.

3. These results imply that in the Anderson model in the localized regime, varying $V(0)$ a little can produce singular spectrum. Indeed, there are disjoint, locally uncountable sets with purely pure point spectrum when $V(0)$ is in one set and pure singular continuous spectrum when $V(0)$ is in the other set!

Another subtle class is the almost-periodic Schrödinger operators. We will consider functions $V$ on $\mathbb{R}$ or $\mathbb{Z}$ that are even and almost periodic (typical examples are $V(n)=\lambda \cos (\pi \alpha n)$ in the $\mathbb{Z}$ case and $V(x)=\lambda \cos (\pi x)+\mu \cos (\pi \alpha x)$ in the $\mathbb{R}$ case with $\alpha$ irrational) and define

$$
\begin{array}{ll}
\mathbb{R} \text { case } & H_{\omega}=-\frac{d^{2}}{d x^{2}}+V_{\omega}(x) \\
\mathbb{Z} \text { case } & \left(H_{\omega} u\right)(n)=u(n+1)+u(n-1)+V_{\omega}(n) u(n)
\end{array}
$$

where $\omega$ is a point in the hull, $\Omega$, of $V$ and $V_{\omega}$ the corresponding potential (in the typical cases above, $\Omega=S^{1}$ and $S^{1} \times S^{1}$ with $V_{\theta}(n)=\lambda \cos (\pi \alpha n+\theta)$ and $\left.V_{\theta, \psi}(x)=\lambda \cos (\pi x+\theta)+\mu \cos (\pi \alpha x+\psi)\right)$. $\Omega$ is a compact metric space in the Bohr topology.

Theorem 6 [23]. Let $V$ be an even almost-periodic potential on $\mathbb{R}$ or $\mathbb{Z}$. Then:

(a) For a dense $G_{\delta}$ in the hull, $H_{\omega}$ has no point spectrum.

(b) If for some point $\omega_{0}$ in the hull $H_{\omega_{0}}$ has no a.c. spectrum, then for a dense $G_{\delta}$ in the hull, $H_{\omega}$ has purely singular continuous spectrum.

Example [23]. In the $\mathbb{Z}$ case, if $V=\lambda \cos (\pi \alpha n+\theta)$ with $\lambda \geq 2$ and $\alpha$ irrational, then it follows that $H_{\theta}$ has purely singular spectrum for a dense $G_{\delta}$ of $\theta$. When $\lambda$ is large $[25,10,16]$, it is known that we have pure point spectrum only for a set of $\theta$ of full Lebesgue measure. Once again, we have locally uncountable sets of parameters with point spectrum for one parameter set and singular continuous spectrum in the other.

\section{REFERENCES}

[1] S. Agmon, Spectral properties of Schrödinger operators and scattering theory, Ann. Scuola Norm. Sup. Pisa Cl. Sci. (4) 2 (1975), 151-218.

[2] M. Aizenman and S. Molchanov, Localization at large disorders and at extreme energies: An elementary derivation, Comm. Math. Phys. 157 (1993), 245-278. 
[3] E. Balslev and J. M. Combes, Spectral properties of many-body Schrödinger operators with dilation-analytic interactions, Comm. Math. Phys. 22 (1971), 280-294.

[4] R. Carmona, Exponential localization in one-dimensional disordered systems, Duke Math. J. 49 (1982), 191-213.

[5] R. del Rio Castillo, A forbidden set for embedded eigenvalues, Proc. Amer. Math. Soc. 121 (1994), 77-82.

[6] R. del Rio, N. Makarov, and B. Simon, Operators with singular continuous spectrum, II. Rank one operators, Comm. Math. Phys. (to appear).

[7] F. Delyon, H. Kunz, and B. Souillard, One-dimensional wave equations in disordered media, J. Phys. A 16 (1983), 25-42.

[8] F. Delyon, Y. Lévy, and B. Souillard, Anderson localization for multi-dimensional systems at large disorder or large energy, Comm. Math. Phys. 100 (1985), 463-470.

[9] J. Fröhlich and T. Spencer, Absence of diffusion in the Anderson tight binding model for large disorder or low energy, Comm. Math. Phys. 88 (1983), 151-189.

[10] J. Fröhlich, T. Spencer, and P. Wittwer, Localization for a class of one dimensional quasiperiodic Schrödinger operators, Comm. Math. Phys. 132 (1990), 5-25.

[11] I. Goldsheid, Asymptotics of the product of random matrices depending on a parameter, Soviet Math. Dokl. 16 (1975), 1375-1379.

[12] I. Goldsheid, S. Molchanov, and L. Pastur, A pure point spectrum of the stochastic onedimensional Schrödinger equation, Funct. Anal. Appl. 11 (1977), 1-10.

[13] A. Gordon, On exceptional value of the boundary phase for the Schrödinger equation of a half-line, Russian Math. Surveys 47 (1992), 260-261.

[14] _ Pure point spectrum under 1-parameter perturbations and instability of Anderson localization, Comm. Math. Phys. (to appear).

[15] P. Halmos, In general a measure preserving transformation is mixing, Ann. Math. 45 (1944), 786-792.

[16] S. Jitomirskaya, Anderson localization for the almost Mathieu equation: A nonperturbative proof, Comm. Math. Phys. (to appear).

[17] T. Kato, Perturbation theory for linear operators (2nd, ed.), Springer-Verlag, Berlin, Heidelberg, and New York, 1980.

[18] S. Kotani and B. Simon, Localization in general one-dimensional random systems, II. Continuum Schrödinger operators, Comm. Math. Phys. 112 (1987), 103-119.

[19] P. Perry, I. Sigal, and B. Simon, Spectral analysis of $N$-body Schrödinger operators, Ann. of Math. (2) 114 (1981), 519-567.

[20] M. Reed and B. Simon, Methods of modern mathematical physics, IV. Analysis of operators, Academic Press, London, 1978.

[21] V. Rohlin, $A$ "general" measure-preserving transformation is not mixing, Dokl. Akad. Nauk SSSR (N.S.) 60 (1948), 349-351.

[22] B. Simon, Operators with singular continuous spectrum, I. General operators, Ann. of Math. (to appear).

[23] B. Simon and S. Jitomirskaya, Operators with singular continuous spectrum, III. Almost periodic Schrödinger operators, Comm. Math. Phys. (to appear).

[24] B. Simon and T. Wolff, Singular continuous spectrum under rank one perturbations and localization for random Hamiltonians, Comm. Pure Appl. Math. 39 (1986), 75-90.

[25] Ya. Sinai, Anderson localization for one-dimensional difference Schrödinger operator with quasiperiodic potential, J. Stat. Phys. 46 (1987), 861-909. 
[26] J. von Neumann, Charakterisierung des Spektrums eines Integraloperators, Actualités Sci. Indust., no. 229, Hermann, Paris, 1935.

[27] H. Weyl, Über beschränkte quadratische Formen, deren Differenz vollstetig ist, Rend. Circ. Mat. Palermo 27 (1909), 373-392.

(R. del Rio, N. Makarov, and B. Simon) Division of Physics, Mathematics and Astronomy, 253-37, California Institute of Technology, Pasadena, California 91125

Current address, R.del Rio: IIMAS-UNAM, Apdo. Postal 20-726, Admon No. 20, 01000 Mexico D.F., Mexico

E-mail address, R. del Rio: delrioeredvax1.dgsca.unam.mx

$E$-mail address, N. Makarov: makarovecaltech.edu

E-mail address, B. Simon: bsimonecaltech.edu

(S. Jitomirskaya) Department of Mathematics, University of California, Irvine, CaliFORNIA 92717

E-mail address: szhitomi@math.uci.edu 